\title{
An Overview of Spectrum Occupancy Models for Cognitive Radio Networks
}

\author{
Miguel López-Benítez and Fernando Casadevall \\ Department of Signal Theory and Communications \\ Universitat Politècnica de Catalunya (UPC) \\ 08034 Barcelona, Spain \\ \{miguel.lopez, ferranc\}@tsc.upc.edu
}

\begin{abstract}
The Dynamic Spectrum Access (DSA) paradigm based on the Cognitive Radio (CR) technology has emerged as a promising solution to conciliate the existing conflicts between spectrum demand growth and current spectrum underutilization without changes to the existing legacy wireless systems. The basic underlying idea of DSA/CR is to allow unlicensed users to access in an opportunistic and non-interfering manner some licensed bands temporarily unused by the licensed users. Due to the opportunistic nature of the DSA/CR paradigm, a realistic and accurate modeling of spectrum occupancy patterns becomes essential in the domain of DSA/CR research. In this context, this paper provides an overview of the existing spectrum occupancy models recently proposed in the literature to characterize the spectrum usage patterns of licensed systems in the time, frequency and space dimensions.
\end{abstract}

Keywords: cognitive radio, dynamic spectrum access, spectrum characterization, spectrum occupancy modeling.

\section{Introduction}

Wireless communication systems have been exploited since the early days of radio communications under a fixed spectrum management policy. Portions of the spectrum separated by guard bands have been allocated to particular licensees over large geographical regions, on a long term basis, and under exclusive exploitation licenses. Under this static regulatory regime, the overwhelming proliferation of new operators, services and wireless technologies has resulted in the depletion of spectrum bands with commercially attractive radio propagation characteristics. As a result, the Dynamic Spectrum Access (DSA) paradigm based on the Cognitive Radio (CR) technology [1] has gained popularity, motivated by the currently inefficient utilization of spectrum already demonstrated by many spectrum measurement campaigns performed all around the world [2, 7, 12 15, 21]. The basic underlying principle of DSA/CR is to allow unlicensed users to access in an opportunistic and non-interfering manner some licensed bands temporarily unoccupied by licensed users. Unlicensed (secondary) DSA/CR terminals monitor the spectrum in order to detect spectrum gaps left 
unused by licensed (primary) users and opportunistically transmit. Secondary unlicensed transmissions are allowed according to this operating principle as long as they do not result in harmful interference to the licensees.

As a result of the opportunistic nature of the DSA/CR principle, the behavior and performance of a network of DSA/CR nodes depends on the primary spectrum occupancy pattern. Realistically and accurately modeling such patterns becomes therefore essential and extremely useful in the domain of DSA/CR research. Models of spectrum use can find applications in a wide variety of fields, ranging from analytical studies to the design, dimensioning and performance evaluation of secondary networks, including the development of innovative simulation tools as well as novel DSA/CR techniques. This paper provides a brief overview of some spectrum occupancy models recently proposed in the literature in the context of DSA/CR research in order to capture and reproduce the statistical properties of spectrum usage. The existing models can be categorized into time-, frequency- and space-dimension models, each of which describe the statistical properties of spectrum usage in the corresponding domain. Based on this classification, this paper reviews some existing modeling approaches and analyzes their merits and limitations.

\section{Time-Dimension Models}

From the point of view of DSA/CR, spectrum usage can adequately be modeled by means of a two-state Markov chain, with one state indicating that the channel is busy and therefore not available for opportunistic access and the other one indicating that the channel is idle and thus available for secondary use. Although some alternative modeling approaches have been proposed in the literature [6], the two-state Markov chain is the most widely employed time-dimension model in DSA/CR research. This binary channel model can be employed to describe the occupancy pattern of a licensed channel in discrete and continuous time.

\subsection{Discrete-Time Models}

In the two-state Discrete-Time Markov Chain (DTMC) model the time index set is discrete. According to this, the channel remains in a given state at each step, with the state changing randomly between steps. The behavior of the DTMC channel model can be described by means of the set of transition probabilities between states (see Figure 1), which can be expressed in matrix form as:

$$
\mathbf{P}=\left[\begin{array}{ll}
p_{00} & p_{01} \\
p_{10} & p_{11}
\end{array}\right]
$$

where $p_{i j}$ represents the probability that the system transitions from state $s_{i}$ to state $s_{j}$. Note that the DTMC channel model in (1), commonly used in the literature, assumes a stationary (time-homogeneous) DTMC, where the transition matrix $\mathbf{P}$ is constant and independent of the time instant. 


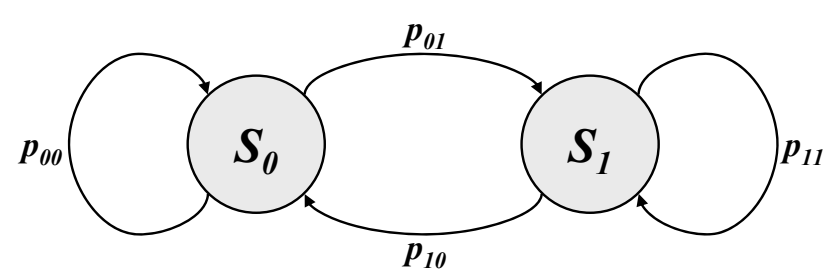

Fig. 1. Discrete-Time Markov Chain (DTMC) model

The mean occupancy level of a channel is certainly a straightforward metric and an accurate reproduction is a minimum requisite for any time-dimension spectrum occupancy model. The average occupancy of a channel can be expressed in terms of the Duty Cycle (DC), henceforth denoted as $\Psi$. The DC of a channel can be defined as the probability (or fraction of time) that the channel is busy (i.e., resides in the busy state in the long term), which for the DTMC model of Figure 1 is given by $\Psi=p_{01} /\left(p_{01}+p_{10}\right)$. The stationary DTMC model of (1) can therefore be configured to reproduce any arbitrary $\Psi$ by selecting the transition probabilities as $p_{01}=p_{11}=\Psi$ and $p_{10}=p_{00}=1-\Psi$, which yields:

$$
\mathbf{P}=\left[\begin{array}{l}
1-\Psi \Psi \\
1-\Psi \Psi
\end{array}\right]
$$

Nevertheless, reproducing not only the mean DC but also the lengths of the busy and idle periods is an important feature of a realistic time-domain model for spectrum use. The stationary DTMC model has been proven to not be able to reproduce the statistical properties of busy and idle period lengths of real channels 9]. This limitation, however, can be overcome by means of a nonstationary (time-inhomogeneous) DTMC channel model with a time-dependent transition matrix:

$$
\mathbf{P}(t)=\left[\begin{array}{l}
1-\Psi(t) \Psi(t) \\
1-\Psi(t) \Psi(t)
\end{array}\right]
$$

In the stationary case of (2), $\Psi$ represents a constant parameter. However, in the non-stationary case of (3),$\Psi(t)$ represents a time-dependent function that needs to be characterized in order to characterize the DTMC channel model in the time domain. The development of mathematical models for $\Psi(t)$ may be relatively simple for primary systems where occupancy patterns are characterized by a strong deterministic component. This is for example the case of cellular mobile communication systems, which exhibit a periodic load variation pattern on a daily basis (see Figure 2). Adequate models for such deterministic patterns have been developed in [9]. In general, however, the traffic load supported by a radio channel is normally the consequence of a significant number of random factors such as the number of incoming and outgoing users and the resource management policies employed in the system. As a result, the channel usage level is itself a random variable that may more appropriately be characterized from a stochastic modeling perspective, which still constitutes an open issue. 


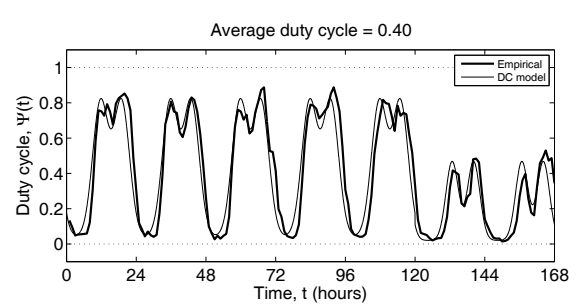

(a)

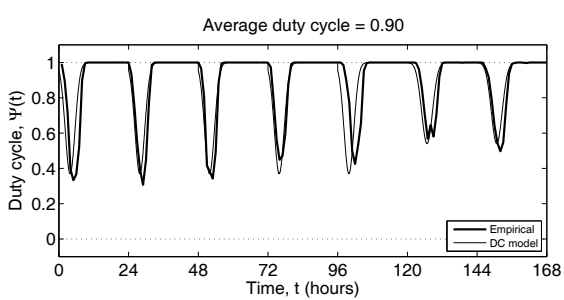

(b)

Fig. 2. Empirical DC time evolution and corresponding deterministic model for: (a) DCS 1800 low/medium-load channel, (b) E-GSM 900 medium/high-load channel

\subsection{Continuous-Time Models}

Another popular model in the DSA/CR literature is the two-state ContinuousTime Markov Chain (CTMC) model, where the channel remains in one state for a random time period before switching to the other state. The state holding time or sojourn time is modeled as an exponentially distributed random variable. Some works based on empirical measurements [3, 5, 16, 18] have demonstrated, however, that state holding times do not follow exponential distributions in practice. In particular, it has been found that state holding times are more adequately described by means of generalized Pareto [4], a mixture of uniform and generalized Pareto [3, 5], hyper-Erlang [3, 5], generalized Pareto and hyper-exponential [16], as well as geometric and log-normal [18] distributions. Based on these results, a more convenient model is therefore the Continuous-Time Semi-Markov Chain (CTSMC) model, where the state holding times can follow any arbitrary distributions. Appropriate parameters for the aforementioned distributions have been derived from field measurements performed with high time-resolution [3, 5, 16. and low time-resolution [18] measurement equipment, and for various radio technologies of practical relevance.

It is worth noting that the CTSMC channel model provides an explicit means to characterize and reproduce the lengths of the busy and idle periods, which implicitly offers the possibility to reproduce any arbitrary DC by appropriately selecting the parameters of the sojourn time distributions in order to provide mean values $\mathbb{E}\left\{T_{i}\right\}$ such that:

$$
\Psi=\frac{\mathbb{E}\left\{T_{1}\right\}}{\mathbb{E}\left\{T_{0}\right\}+\mathbb{E}\left\{T_{1}\right\}}
$$

where $\mathbb{E}\left\{T_{0}\right\}$ and $\mathbb{E}\left\{T_{1}\right\}$ represent the mean sojourn times in the idle and busy states (i.e., mean idle and busy period duration), respectively.

\subsection{Time-Correlation Models}

The DTMC (with appropriate DC models) and CTSMC channel models are capable to capture and reproduce the mean channel occupancy and the statistical 
distributions of busy and idle periods observed in real channels. Nevertheless, previous studies [10, 18] have indicated that in some cases the lengths of the busy and idle periods can be correlated, a feature that the DTMC and CTSMC models cannot reproduce. Although in general high correlation levels are not observed in practice, an accurate and realistic model of spectrum usage should take this feature into account.

Experimental studies 10] have shown that the lengths of busy and idle periods exhibit negative correlation coefficients, meaning that when the length of a busy period increases, the length of the next idle period tends to decrease and vice versa. This can be explained by the fact that when the channel load increases, then the fraction of time that it remains in use increases and, as a result, the duration of busy periods increases while idle periods become shorter. On the other hand, the opposite behavior is observed when the channel load decreases (i.e., the length of busy periods decreases and idle periods become longer). The correlation between the sequence of periods of the same type (either busy or idle) of a channel and a shifted version of itself (i.e., the autocorrelation) has experimentally been observed to exhibit two different behaviors, namely one periodic and another non-periodic. This is illustrated in Figure 3, which shows some examples of the autocorrelation function of idle periods as a function of the lag number, $m$, based on the Spearman's rank correlation coefficient, which is denoted as $\rho_{s}\left(T_{0}, T_{0} ; m\right)$.

For channels with periodic autocorrelation functions (see Figure 3(a)] with period $M$, the correlation coefficient can be expressed as the summation of two bell-shaped exponential terms centered at lags $m=1$ and $m=M+1$, with amplitudes $A$ and widths $\sigma$ :

$$
\rho_{s}\left(T_{i}, T_{i} ; m\right)= \begin{cases}1, & m=0 \\ \rho_{s}^{\text {min }}+A e^{-\left(\frac{m-1}{\sigma}\right)^{2}}+A e^{-\left(\frac{m-M-1}{\sigma}\right)^{2}}, & 1 \leq m \leq M\end{cases}
$$

where $\rho_{s}^{\text {min }}$ is the minimum correlation. Based on field measurements, it has empirically been observed that $\rho_{s}^{\text {min }} \approx-0.1$ in most cases, $A \in[0.2,0.5], M$ corresponds to the average number of lags equivalent to 24 hours, and $\sigma \approx M / 4$.

For channels with non-periodic autocorrelation functions (see Figure 3(b)], the correlation coefficient takes its maximum value $\rho_{s}^{\max }$ at $m=1$ and decreases linearly with $m$ until $m=M$, beyond which the correlation is approximately zero. This behavior can adequately be modeled as:

$$
\rho_{s}\left(T_{i}, T_{i} ; m\right)= \begin{cases}1, & m=0 \\ \rho_{s}^{\max }\left(\frac{M-m}{M-1}\right), & 1 \leq m \leq M \\ 0, & m>M\end{cases}
$$

In this case, it has been observed that $\rho_{s}^{\max } \in[0.1,0.4]$ and $M \in[2000,8000]$.

As it can clearly be appreciated in Figure 3, the models of (5) and (6) are able to accurately describe the time-domain autocorrelation properties of spectrum usage empirically observed in real systems. 


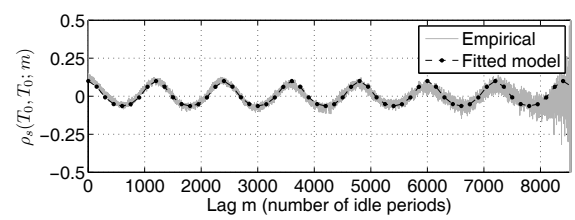

(a)

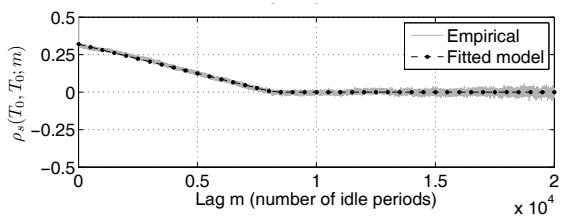

(b)

Fig. 3. Spearman's rank autocorrelation function of idle periods for: (a) periodic pattern, (b) non-periodic pattern

To reproduce time correlations, a simulation approach based on the aggregation and superposition of the realizations of several CTSMC processes has been proposed in [19]. The main limitation of such proposal is that the resulting correlations depend on the number of aggregated processes as well as their distributions, and the distribution parameters as well as the number of processes to be aggregated in order to reproduce a particular correlation level cannot easily be determined, making necessary the use of simulations to this end, which complicates the configuration of the model and hence its application. An alternative approach based on random variate generation principles has been proposed in [10]. Such another proposal requires as input information the particular distributions of idle and busy periods to be reproduced, the desired correlation coefficient between busy/idle periods as well as the desired autocorrelation function (periodic or non-periodic) of idle or busy periods. The algorithm outputs sequences of busy/idle period durations satisfying the desired specifications.

\section{Frequency-Dimension Models}

The previous section has reviewed existing models to describe the statistical properties of spectrum occupancy patterns on individual channels. Such modeling approaches can be extended by considering as a whole the channels belonging to the same allocated band and introducing additional models to describe the statistical properties of spectrum usage in the frequency dimension.

In the spectral domain, one of the simplest but probably most relevant aspects to be captured and reproduced is the statistical distribution of the channel occupancy levels. The DCs of individual channels belonging to the same band have been shown to follow a beta distribution [17], whose density function is:

$$
f_{x}^{B}(x ; \alpha, \beta)=\frac{1}{B(\alpha, \beta)} x^{\alpha-1}(1-x)^{\beta-1}, \quad x \in(0,1)
$$

where $\alpha>0$ and $\beta>0$ are shape parameters and $B(\alpha, \beta)$ is the beta function. Alternatively, the Kumaraswamy distribution can be used as well:

$$
f_{x}^{K}(x ; a, b)=a b x^{a-1}\left(1-x^{a}\right)^{b-1}, \quad x \in(0,1)
$$

where $a>0$ and $b>0$ are shape parameters. The Kumaraswamy distribution is similar to the beta distribution, but easier to use in analytical studies due to the 


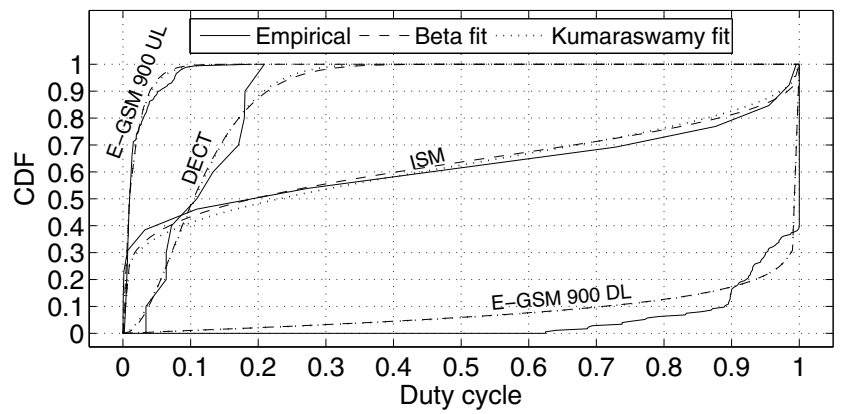

Fig. 4. Empirical DC distributions and corresponding beta and Kumaraswamy fits

simpler form of its density function. Figure 4 shows some examples of empirical DC distributions and their corresponding beta and Kumaraswamy fits.

Both distributions can be configured to reproduce any arbitrary mean DC over the whole band, $\bar{\Psi}$, by properly selecting the shape parameters:

$$
\bar{\Psi}=\frac{\alpha}{\alpha+\beta}=b B\left(1+\frac{1}{a}, b\right)
$$

\section{Space-Dimension Models}

While time- and frequency-dimension models are intended to reproduce the statistical properties of real spectrum occupancy patterns of primary transmitters, space-dimension models in general deal with the characterization of spectrum occupancy patterns as perceived by the DSA/CR users at various locations.

Spectrum occupancy in the space domain is analyzed and characterized in [20] in terms of the spatial distribution of the Power Spectral Density (PSD) by means of spatial statistics and random fields. Concretely, a semivariogram analytical model is fitted to average PSD values obtained at various locations by means of field measurements (empirical model) and simulation tools (deterministic model). The resulting PSD values can be mapped to binary busy/idle perceptions at various locations by means of a thresholding technique.

An alternative probabilistic modeling approach has been developed in [8, 11], which is illustrated in Figure 5. In the first step, a radio propagation model is used to estimate, based on a set of input parameters $\mathbf{p}=\left(p_{1}, p_{2}, \ldots, p_{M}\right)$ such as operating frequency, distance, etc., the radio propagation loss $L$ between the primary transmitter and the DSA/CR user. Based on the primary transmission power $P_{T}$, the computed losses $L$ are then employed to compute the primary power $P_{S}$ that would be observed by DSA/CR nodes at various locations, which are translated to SNR values $\gamma$ based on the receiver's noise power $P_{N}$. The resulting SNR values are then fed, along with an additional set of input 


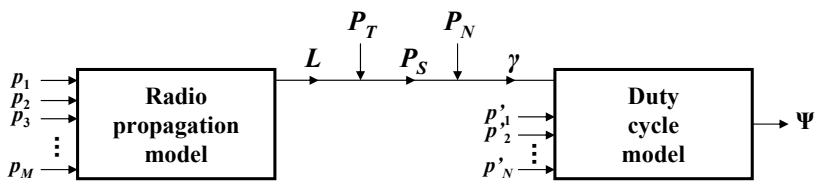

Fig. 5. Spatial spectrum occupancy modeling approach [8, 11]

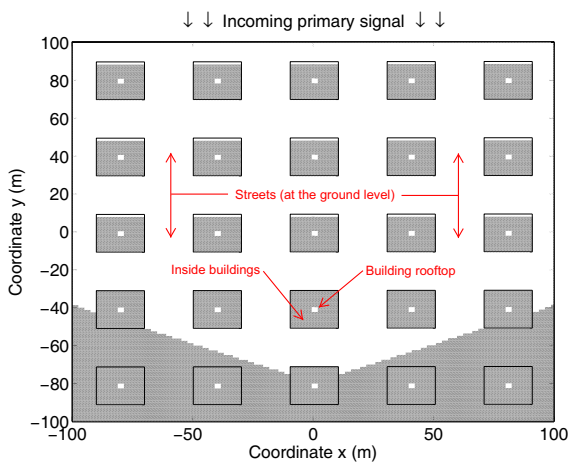

(a)

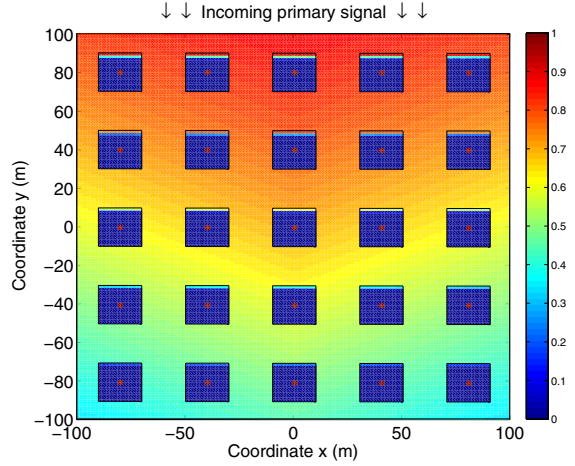

(b)

Fig. 6. Characterization of spatial spectrum occupancy perception at various locations in a realistic urban environment:(a) in terms of binary busy (white)/idle (gray) observations, (b) in terms of the probability to observe spectrum as busy

parameters $\mathbf{p}^{\prime}=\left(p_{1}^{\prime}, p_{2}^{\prime}, \ldots, p_{N}^{\prime}\right)$, to a DC model, which outputs an estimation $\Psi$ of the DC that would be perceived at the considered geographical locations [8]:

$$
\Psi=\left(1-\sum_{k=1}^{K} \alpha_{k}\right) P_{f a}+\sum_{k=1}^{K} \alpha_{k} Q\left(\frac{Q^{-1}\left(P_{f a}\right) \sigma_{N}-\gamma_{k}}{\sigma_{S_{k}}}\right)
$$

where $K>0$ represents the number of transmission power levels that may be present in the channel, $0<\alpha_{k} \leq 1$ is the activity factor of the $k$-th power level, $P_{f a}$ is the target probability of false alarm of the DSA/CR network, $\gamma_{k}(\mathrm{~dB})=$ $P_{S_{k}}(\mathrm{dBm})-P_{N}(\mathrm{dBm})$ is the SNR resulting from the $k$-th average transmission power level, and $\sigma_{S_{k}}, \sigma_{N}$ are the standard deviation of the $k$-th signal and noise power levels respectively. These parameters constitute the input vector $\mathbf{p}^{\prime}$.

Figure 6 shows an example of the spectrum occupancy perceived by DSA/CR users at various locations in a realistic urban environment at the ground level between buildings, inside buildings and at rooftops [11]. While PSD/power modeling methods combined with thresholding techniques provide a simple binary characterization of the spatial spectrum occupancy perception (Figure 6(a), the approach depicted in Figure 5 provides a more sophisticated characterization (Figure 6(b) by means of the probability that the spectrum is observed as busy depending on the specific DSA/CR user location, the considered scenario and the surrounding radio propagation environment. 


\section{Conclusions}

The development of the DSA/CR technology can significantly benefit from accurate and practical spectrum usage models. The purpose of such models is to provide a tractable, yet realistic representation of the statistical properties of spectrum usage in real systems that can adequately be employed in analytical studies or implemented in simulation tools for the performance evaluation of DSA/CR techniques. In this context, this paper has provided an overview of spectrum occupancy models recently proposed in the literature in the context of DSA/CR. The existing models can be broadly categorized into time-, frequency- and space-dimension models. Based on this classification, this paper has reviewed various modeling approaches, pointing out their advantages and shortcomings.

Acknowledgments. This work was supported by the European Commission in the framework of the FP7 FARAMIR Project (Ref. ICT-248351) and the Spanish Research Council under research project ARCO (Ref. TEC2010-15198). The support from the Spanish Ministry of Science and Innovation (MICINN) under FPU grant AP2006-848 is hereby acknowledged.

\section{References}

1. Akyildiz, I.F., Lee, W.-Y., Vuran, M.C., Mohanty, S.: NeXt generation/dynamic spectrum access/cognitive radio wireless networks: a survey. Computer Networks 50(13), 2127-2159 (2006)

2. Chiang, R.I.C., Rowe, G.B., Sowerby, K.W.: A quantitative analysis of spectral occupancy measurements for cognitive radio. In: Proc. IEEE 65th Vehicular Tech. Conf. (VTC 2007 Spring), pp. 3016-3020 (April 2007)

3. Geirhofer, S., Tong, L., Sadler, B.M.: Dynamic spectrum access in WLAN channels: Empirical model and its stochastic analysis. In: Proc. First Int'l. Workshop on Tech. and Policy for Accessing Spectrum (TAPAS 2006), pp. 1-10 (August 2006)

4. Geirhofer, S., Tong, L., Sadler, B.M.: A measurement-based model for dynamic spectrum access in WLAN channels. In: Proc. IEEE Military Comms. Conf. (MILCOM 2006), pp. 1-7 (October 2006)

5. Geirhofer, S., Tong, L., Sadler, B.M.: Dynamic spectrum access in the time domain: Modeling and exploiting white space. IEEE Comms. Magazine 45(5), 66-72 (2007)

6. Ghosh, C., Pagadarai, S., Agrawal, D.P., Wyglinski, A.M.: A framework for statistical wireless spectrum occupancy modeling. IEEE Trans. on Wireless Comms. 9(1), 38-55 (2010)

7. Islam, M.H., et al.: Spectrum survey in Singapore: Occupancy measurements and analyses. In: Proc. 3rd Int'l. Conf. on Cognitive Radio Oriented Wireless Networks and Comms. (CrownCom 2008), pp. 1-7 (May 2008)

8. López-Benítez, M., Casadevall, F.: Spatial duty cycle model for cognitive radio. In: Proc. 21st Annual IEEE Int'l. Symp. on Personal, Indoor and Mobile Radio Comms. (PIMRC 2010), pp. 1631-1636 (September 2010)

9. López-Benítez, M., Casadevall, F.: Discrete-time spectrum occupancy model based on markov chain and duty cycle models. In: Proc. 5th Int'l. Symp. on Dynamic Spectrum Access Networks (DySPAN 2011), pp. 1-10 (May 2011) 
10. López-Benítez, M., Casadevall, F.: Modeling and simulation of time-correlation properties of spectrum use in cognitive radio. In: Proc. 6th Int'l. ICST Conf. on Cognitive Radio Oriented Wireless Networks (CrownCom 2011), pp. 1-5 (June 2011)

11. López-Benítez, M., Casadevall, F.: Statistical prediction of spectrum occupancy perception in dynamic spectrum access networks. In: Proc. IEEE Int'l. Conf. on Comms. (ICC 2011), pp. 1-6 (June 2011)

12. López-Benítez, M., Casadevall, F., Umbert, A., Pérez-Romero, J., Palicot, J., Moy, C., Hachemani, R.: Spectral occupation measurements and blind standard recognition sensor for cognitive radio networks. In: Proc. 4th Int'l. Conf. on Cognitive Radio Oriented Wireless Networks and Comms. (CrownCom 2009), pp. 1-9 (June 2009)

13. McHenry, M.A., et al.: Spectrum occupancy measurements. Tech. rep., Shared Spectrum Company (January 2004-August 2005)

14. Petrin, A., Steffes, P.G.: Analysis and comparison of spectrum measurements performed in urban and rural areas to determine the total amount of spectrum usage. In: Proc. Int'l. Symp. on Advanced Radio Techs. (ISART 2005), pp. 9-12 (March 2005)

15. Schiphorst, R., Slump, C.H.: Evaluation of spectrum occupancy in Amsterdam using mobile monitoring vehicles. In: Proc. IEEE 71st Vehicular Tech. Conf. (VTC Spring 2010), pp. 1-5 (May 2010)

16. Stabellini, L.: Quantifying and modeling spectrum opportunities in a real wireless environment. In: Proc. IEEE Wireless Comms. and Networking Conf. (WCNC 2010), pp. 1-6 (April 2010)

17. Wellens, M., Mähönen, P.: Lessons learned from an extensive spectrum occupancy measurement campaign and a stochastic duty cycle model. Mobile Networks and Applications 15(3), 461-474 (2010)

18. Wellens, M., Riihijärvi, J., Mähönen, P.: Empirical time and frequency domain models of spectrum use. Physical Comm. 2(1-2), 10-32 (2009)

19. Wellens, M., Riihijärvi, J., Mähönen, P.: Modelling primary system activity in dynamic spectrum access networks by aggregated ON/OFF-processes. In: Proc. Fourth IEEE Workshop on Networking Techs. for Software Defined Radio Networks (SDR 2009), pp. 1-6 (June 2009)

20. Wellens, M., Riihijärvi, J., Mähönen, P.: Spatial statistics and models of spectrum use. Computer Comms. 32, 1998-2001 (2009)

21. Wellens, M., Wu, J., Mähönen, P.: Evaluation of spectrum occupancy in indoor and outdoor scenario in the context of cognitive radio. In: Proc. Second Int'l. Conf. on Cognitive Radio Oriented Wireless Networks and Comms. (CrownCom 2007), pp. 1-8 (August 2007) 